\title{
Microwave assisted extraction of mangiferin from Curcuma amada
}

\author{
K. Padmapriya $\cdot$ Abhishek Dutta \\ Surabhi Chaudhuri $\cdot$ Debjani Dutta
}

Received: 21 July 2011/Accepted: 23 August 2011/Published online: 10 September 2011

(C) The Author(s) 2011. This article is published with open access at Springerlink.com

\begin{abstract}
Mangiferin present in Curcuma amada was extracted with the help of microwave assisted extraction (MAE). The extraction solvent used was ethanol, which is eco-friendly and reduced the risk of environmental hazard. The mangiferin content was found to increase until $500 \mathrm{~W}$, but decreased as the microwave power was increased further. A similar threshold was also obtained for microwave irradiation time. Following a mathematical analysis, an optimal mangiferin yield of $41 \mu \mathrm{g} / \mathrm{mL}$ was obtained from an extraction time of $15.32 \mathrm{~s}$ for a microwave power of $500 \mathrm{~W}$.
\end{abstract}

Keywords Mangiferin - Microwave power .

Microwave irradiation time $\cdot$ Mathematical model

\section{Introduction}

Due to the presence of health promoting components such as vitamins, minerals, antioxidants and prebiotics, there is a growing interest in the extraction of the bioactive compounds from fruits, vegetables and spices. Several techniques are available for the extraction of bioactive compounds from plant sources including Soxhlet, microwave assisted extraction (MAE), ultrasound-assisted extraction (UAE), and heat reflux extraction (HRE). The

K. Padmapriya $\cdot$ S. Chaudhuri · D. Dutta $(\bowtie)$

Department of Biotechnology, National Institute of Technology, Durgapur 713209, India

e-mail: debs_2000in@yahoo.com; debjani.dutta@bt.nitdgp.ac.in

\section{A. Dutta}

BIOMATH, Department of Mathematical Modelling,

Statistics and Bioinformatics, Ghent University,

Coupure Links 653, 9000 Ghent, Belgium main advantages of MAE are the considerable reduction in time and solvent as compared to the conventional techniques (Xiao et al. 2008). Curcuma amada, an understudied spice, belongs to the family Zingiberaceae, and has medicinal properties. It looks like ginger, but has mango flavour and is usually used for culinary purposes in the Indian sub-continent. Mangiferin is a phytochemical and is said to be present in mango ginger. It has a wide range of pharmacological activities including anti-diabetic, antiHIV, anti-cancer, immune modulatory, anti-inflammatory and anti-oxidant properties (Wauthoz et al. 2007). However, no reports on MAE of mangiferin from C. amada have yet been published. In this study, we report on the feasibility of MAE for the extraction of mangiferin from C. amada. Our work aims to determine the effect of different microwave power and irradiation time on the mangiferin extraction from C. amada along with the kinetic parameters for efficient extraction through simple mathematical approach.

\section{Materials and methods}

Mango ginger (C. amada) was purchased from a local supplier in Durgapur, India. The rhizomes were washed, sliced and dried in a hot air oven (OVFU, India) at $60{ }^{\circ} \mathrm{C}$ for $72 \mathrm{~h}$ and powdered in a grinder. Mangiferin standard was purchased from Sigma Aldrich, USA. The extraction of mangiferin was carried out with the method of Krivut et al. (1985) with slight modifications. The C. amada extract obtained after microwave irradiation was evaporated in a Rotary Vacuum Evaporator (Yamato, Japan), filtered and separated to remove other inactive molecules using chloroform and butanol. The final purified extract was evaporated and dissolved in DMSO and analysed with

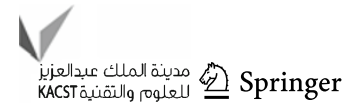


a UV-vis spectrophotometer (UV-2310, Techcomp), at $410 \mathrm{~nm}$. The estimation of mangiferin was followed using the method described by Joubert et al. 2008.

\section{Statistical analysis}

The statistical software SPSS 11 (SPSS, Chicago, IL) was used for the data analysis. The two factors, microwave power (MP, main factor) and microwave irradiation time (MIT, random factor), were treated on a randomised complete block design. Means and coefficients of variance were computed for all qualitative analysis and treatments with homogeneous means ranked using the Newman-Keuls post hoc test. The effect of these two factors with mangiferin content was compared using the bi-variant correlation analysis. A paired sample $T$ test was used to compare the differences between two groups. The significance of the results was established at values greater than 0.05 in all the experiments performed.

\section{Result and discussion}

Effect of microwave power and irradiation time on the mangiferin yield

$2.5 \mathrm{gm}$ of dried powder of $C$. amada was extracted with $50 \mathrm{~mL}$ of $50 \%$ aqueous ethanol under different microwave power $(350,450,500$ and $600 \mathrm{~W})$ for different MIT (5-80 s), as shown in Fig. 1. The solvent used was $50 \%$ ethanol, as the solvent of medium polarity was found to improve mangiferin extraction (unpublished results). The concentration of mangiferin and the extraction efficiency were found to improve when the microwave power was raised from 350 to $500 \mathrm{~W}$. When the microwave power was raised from 350 to $500 \mathrm{~W}$, electromagnetic energy was transferred to the extraction system quickly and this improved the extraction efficiency. However, the concentration of mangiferin decreased at $600 \mathrm{~W}$ microwave power. Similar results were observed by Xiao et al. (2008) in the extraction of flavonoid from Radix astragali at high power due to non-orderly molecular interactions. During shorter irradiation time (till $20 \mathrm{~s}$ ), the mangiferin content increased with microwave power. However, when the extraction solution was heated beyond $20 \mathrm{~s}$ the mangiferin content reduced, and this reduction was observed in all microwave powers. This might be due to the high temperature involved in the extraction process which might have caused degradation of mangiferin (Kim et al. 2010). The mangiferin content was $24 \mu \mathrm{g} / \mathrm{mL}, 35.26 \mu \mathrm{g} / \mathrm{mL}$ and $41.10 \mu \mathrm{g} / \mathrm{mL}$ at $350 \mathrm{~W}, 450 \mathrm{~W}$ and $500 \mathrm{~W}$, respectively, with an exposure of 20-s irradiation time which decreased to $3.083 \mu \mathrm{g} / \mathrm{mL}, 4.35 \mu \mathrm{g} / \mathrm{mL}$ and $5.87 \mu \mathrm{g} / \mathrm{mL}$ after $80 \mathrm{~s}$ of MIT. This result indicates that very high irradiation time is not suitable for mangiferin extraction.

Statistical results indicate that the mangiferin concentration is negatively correlated with both microwave power (insignificant, $p=0.721$ ) and MIT (significant, $p=0.000$ ). The paired sample $T$ test is significant between the two groups, i.e. mangiferin concentration with MIT and microwave power (MP). The results of analysis of variance
Fig. 1 Effect of microwave power (350-600 W) and microwave irradiation time (5-80 s) on the mangiferin concentration

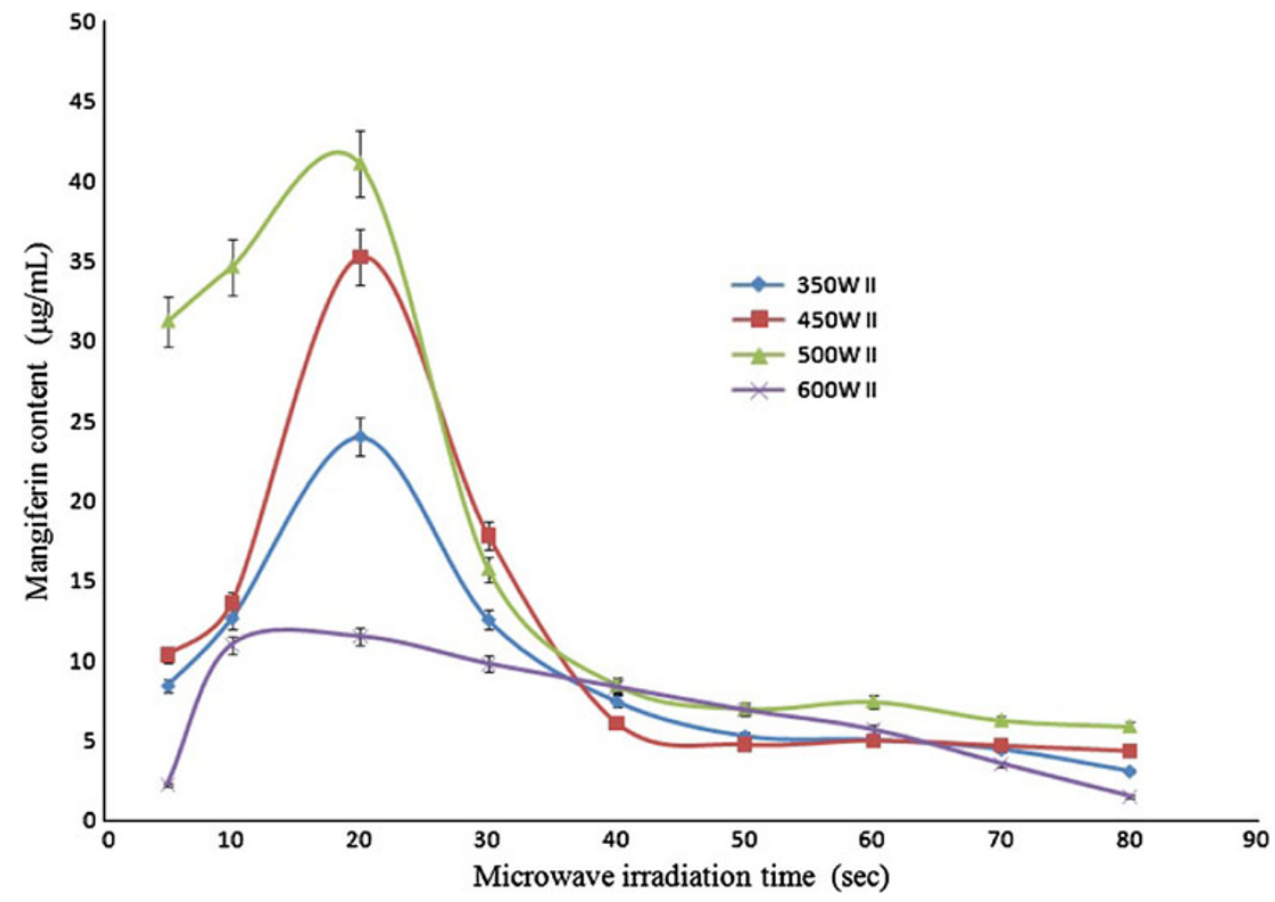


(ANOVA) are given in Table 1. Both microwave power and MIT along with their interaction is significantly different. Newman-Keuls test shows that the mangiferin content is significantly different at all microwave power, with microwave power of $500 \mathrm{~W}$ yielding the highest mean mangiferin content. In case of MIT, there is no significant difference in mangiferin content at 50 and 60 s. However, the mangiferin content differ significantly with remaining MIT. Statistical results indicate that the optimal mangiferin yield was obtained at $500 \mathrm{~W}$ microwave power and irradiation time of around $20 \mathrm{~s}$.

Mathematical model formulation

Considering the two process parameters namely, the MIT and microwave power, an extraction model describing the relationship between these parameters and the extraction efficiency was constructed. The parameters of the model were fitted with a non-linear least-squares (NLLS) Marquardt-Levenberg algorithm, using the device-independent plotting program Gnuplot. It must be noted that the meanaveraged data used in the mathematical modelling is obtained by repeating the extraction process using different samples of $C$. amada. The response for $600 \mathrm{~W}$ was found to be different from the other three responses (i.e. from 350,450 and $500 \mathrm{~W}$ ) for all the samples studied, and did not follow a clear sequence. The same had been observed for the response at $600 \mathrm{~W}$ microwave power using statistical analysis. The dataset of $600 \mathrm{~W}$ was, therefore, neglected while validating the model. This assumption is further strengthened by the statistical analysis, mentioned in the previous section, that a probable optimum can be found at $500 \mathrm{~W}$ and somewhere around $20 \mathrm{~s}$ of irradiation time. Based on the experimental data (see Fig. 1), the following expression was formulated:

$f(x, y)=a_{0}\left(y-a_{1}\right)\left(x / a_{2}\right)^{a_{3} \exp \left(-x / a_{2}\right)+\left(y-a_{7}\right) / a_{6}}$,

where $x$ and $y$ denotes irradiation time (in s) and microwave power (in W), respectively. Equation (1) was obtained by presuming one term constant in time (evolving a little with power since the time evolutions do not reach zero but to some positive limit), and the other term (which is the product of exponential decay in time with a certain power in time) modulated linearly by power. The optimal fit (in green) of the experimental data (in red) using the mathematical model is shown in Fig. 2. The optimal values of the parameters obtained due to regression are given in Table 2 .

Table 1 Results of a one-way ANOVA showing the significance of the two sources (MIT and MP) on the dependent variable (mangiferin concentration) $\left(R^{2}=1\right.$, adjusted $\left.R^{2}=0.999\right)$

\begin{tabular}{llcccc}
\hline Source & Degree of freedom & Type III sum of squares & Mean square & $F$ value & Significance \\
\hline Microwave power (MP) & 1122.190 & 3 & 374.063 & 5269.203 & 0.000 \\
Microwave irradiation time (MIT) & 3990.157 & 8 & 498.770 & 7025.866 & 0.000 \\
MP $\times$ MIT & 1680.888 & 24 & 70.037 & 986.569 \\
Error & 2.556 & 36 & $7.099 \mathrm{E}-02$ & 0.000 \\
Corrected total & 6795.791 & 71 & & \\
\hline
\end{tabular}

Fig. 2 Proposed mathematical model (in green) for the microwave extraction procedure using the experimental data (in red) indicating optimal fit

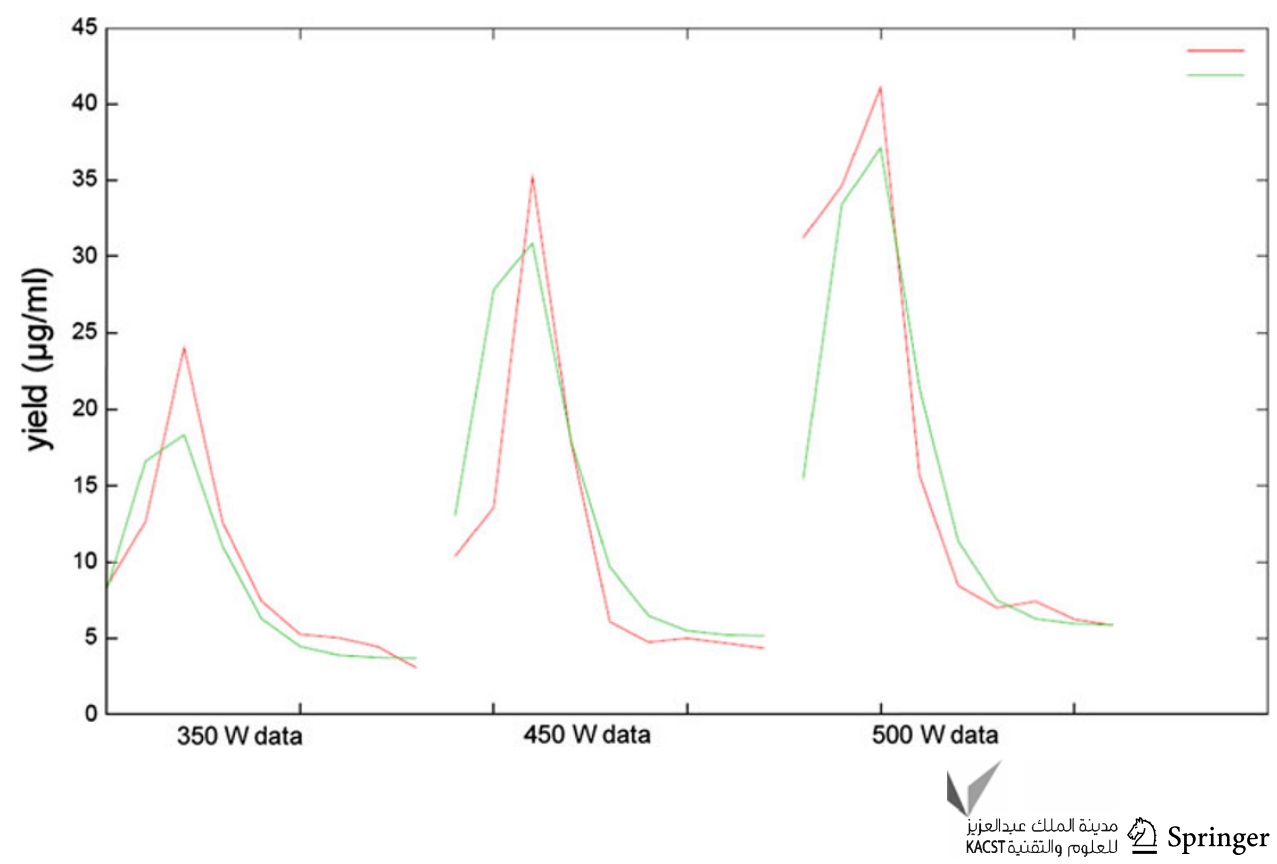


Table 2 Parameter values optimised using non-linear least-squares (NLSS) method

\begin{tabular}{lll}
\hline Parameters & Values & Asymptotic SE $( \pm)$ \\
\hline$a_{0}$ & 0.106 & 0.1024 \\
$a_{1}$ & 217.723 & 92.32 \\
$a_{2}$ & 5.3754 & 1.59 \\
$a_{3}$ & 2.8665 & 0.8596 \\
$a_{6}$ & 69.222 & 109.5 \\
$a_{7}$ & 93.221 & 534.9 \\
\hline
\end{tabular}

Using the mathematical model, an optimal yield of $40.65 \mu \mathrm{g} / \mathrm{mL}$ was obtained from an irradiation time of $15.32 \mathrm{~s}$ for a microwave power of $500 \mathrm{~W}$. The root-meansquare (RMS) value of residuals and the variance of residuals (reduced Chi-square) was calculated to be 5.38 and 28.98 based on 21 degrees of freedom, respectively. Note that the tolerance value set for the parameter estimation is $1 \times 10^{-5}$. The predicted model fitted well with the experimental data. It can be concluded that the proposed model correctly describes the MAE of mangiferin from $C$. amada. However, to obtain a more accurate fit, it is necessary to use additional process parameters for the mathematical model such as solvent mass ratio, solvent concentration, extraction cycle and extraction (leaching) time. These factors have been found to influence mangiferin yield while using traditional extraction techniques.

\section{Conclusion}

This study demonstrates the feasibility of microwave assisted extraction of mangiferin from $C$. amada. Experimental results showed that the mangiferin content initially increased up to $20 \mathrm{~s}$ of irradiation time for all microwave power studied. A statistical analysis was performed which indicated an optimum mangiferin concentration at $500 \mathrm{~W}$ for an irradiation time of around $20 \mathrm{~s}$. Optimal values of irradiation time and microwave power was obtained using a mathematical model based on nonlinear least-squares technique. Moreover, usage of ethanol as an eco-friendly alternative overcomes the serious hazards posed by various techniques adapted by humans. Thus, the extraction of mangiferin from $C$. amada using microwaves and ethanol is efficient not only from the industrial point of view by preventing environmental hazards, but also can be controlled using simple mathematical model. However, a rigorous experimental analysis using several influencing factors needs to be performed which can help posit the mathematical relationship justified by the mechanisms involved.

Acknowledgments One of the authors (AD) would like to thank Dr. Denis Constales, Vakgroep Wiskundige Analyse, Ghent University, Belgium for fruitful discussions related to the mathematical modelling.

Open Access This article is distributed under the terms of the Creative Commons Attribution License which permits any use, distribution and reproduction in any medium, provided the original author(s) and source are credited.

\section{References}

Joubert E, Manley M, Botha M (2008) Evaluation of spectrophotometric methods for screening of green rooibos (Aspalathus linearis) and green honeybush (Cyclopia genistoides) extracts for high levels of bio-active compounds. Phytochem Anal 19:169-178

Kim WJ, Veriansyah B, Lee YW, Kim J, Kim JD (2010) Extraction of mangiferin from Mahkota Dewa (Phaleria macrocarpa) using subcritical water. J Ind Eng Chem 16:425-430

Krivut BA, Fedyunina NA, Kocherga SI, Rusakova SV (1985) Process for preparing mangiferin. United States Patent, Patent number 4518592

Wauthoz N, Balde A, Balde ES, Damme MV, Duez P (2007) Ethnopharmacology of Mangifera indica L bark and pharmacological studies of its main glucosylxanthone, mangiferin. Int $\mathbf{J}$ Biomed Pharma Sci 1(2):112-119

Xiao W, Han L, Shi B (2008) Microwave-assisted extraction of flavonoids from Radix astragali. Sep Purif Technol 62:614-618 